\title{
Transmission Asymmetry in Nano-opto-mechanical Metamaterials
}

\author{
Jinxiang $\mathrm{Li}^{1^{*}}$, Kevin F. MacDonald ${ }^{1}$, and Nikolay I. Zheludev ${ }^{1,2}$ \\ ${ }^{1}$ Optoelectronics Research Centre and Centre for Photonic Metamaterials, \\ University of Southampton, Highfield, Southampton, SO17 1BJ, UK \\ ${ }^{2}$ Centre for Disruptive Photonic Technologies, School of Physical and Mathematical Sciences and The \\ Photonics Institute, Nanyang Technological University Singapore, 637378, Singapore \\ *jinxiang.li@soton.ac.uk
}

We report on the manifestation of strong transmission asymmetry in nano-opto-mechanically reconfigurable all-dielectric metamaterials.

In planar photonic metamaterials manufactured on free-standing elastic nano-membranes the geometric conformation of metamolecules can be reversibly reconfigured by external stimuli to modulate optical properties at high (potentially GHz) frequencies. Nanomechanical metamaterials can be engineered to exhibit profound electro-, magneto- and acousto-optic switching coefficients; to present large effective optical nonlinearities; and to enable the exploration/exploitation of optical phenomena that are extremely small, rare or non-existent in bulk optical media.

Here, we show that resonantly enhanced optical forces in free-standing photonic metamaterials can be comparable in magnitude to the elastic restoring forces resulting from nanoscale deformations in such structures. These forces can be engaged to dynamically reconfigure the constituent cells of the metamaterial in a manner that depends upon the direction of light propagation, giving rise to transmission asymmetry potentially exceeding $60 \%$ in structure less than one third of a wavelength thick at low $\left(<200 \mu \mathrm{W} / \mu \mathrm{m}^{2}\right)$ intensities, as illustrated in Fig. 1.

We present the first experimental evidence for observation of the phenomenon and computationally analyse its underpinnings in differential mode conversion for opposing propagation directions.

(a)

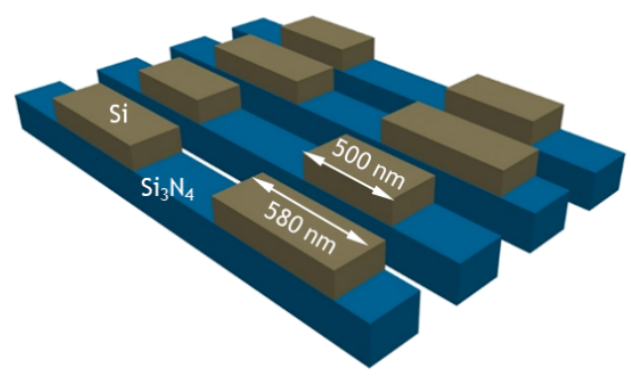

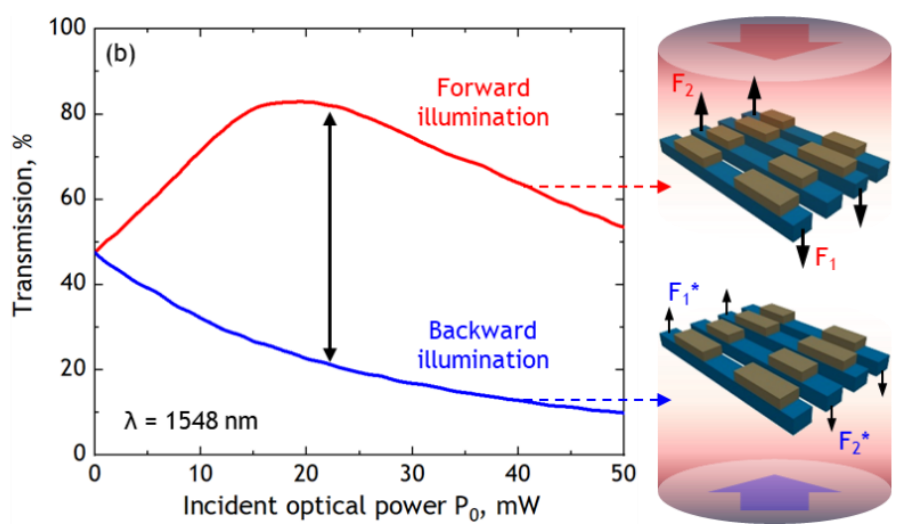

Fig. 1: (a) Schematic illustration of all-dielectric metamaterial manifesting optomechanical asymmetric transmission, comprised of alternately long and short [115 $\mathrm{nm}$ thick] silicon nano-bricks on free-standing [300 $\mathrm{nm}$ thick, $250 \mathrm{~nm}$ wide] silicon nitride beams. (b) Near-infrared transmission of the metamaterial as a function of incident power [on an array of $12 \times 12$ unit cells] for forward and backward directions of illumination. Illumination direction-dependent mutual out-of-plane displacement of the beams induced by resonantly enhanced optical forces lead to strong transmission asymmetry. 